\title{
Optimal Sensor Placement for Predictive Cardiac Motion Modeling
}

\author{
Qian Wu, Adrian J. Chung, and Guang-Zhong Yang \\ Department of Computing, Imperial College London \\ \{q.wu, a.chung, g.z.yang\}@imperial.ac.uk \\ http://vip.doc.ic.ac.uk
}

\begin{abstract}
Subject-specific physiological motion modeling combined with lowdimensional real-time sensing can provide effective prediction of acyclic tissue deformation particularly due to respiration. However, real-time sensing signals used for predictive motion modeling can be strongly coupled with each other but poorly correlated with respiratory induced cardiac deformation. This paper explores a systematic framework based on sequential feature selection for optimal sensor placement so as to achieve maximal model sensitivity and prediction accuracy in response to the entire range of tissue deformation. The proposed framework effectively resolves the problem encountered by traditional regression methods in that the latent variables from both the input and output of the regression model are used to establish their inner relationships. Detailed numerical analysis and in vivo results are provided, which demonstrate the potential clinical value of the technique.
\end{abstract}

\section{Introduction}

The management of acyclic tissue deformation due to voluntary and involuntary motion of the patient plays an important role in high-resolution imaging, intensity modulated radiation therapy, and intra-operative guidance. Despite the steady advances in imaging techniques, in situ motion adaptation based on real-time 3D imaging remains a challenge, largely due to the prohibitive bandwidth and processing requirements. For this reason, current research is focused on using a priori subjectspecific physiological motion modeling combined with low-dimensional real-time sensing to provide effective deformation prediction. In cardiovascular MR, for example, the use of diaphragmatic navigators is now a popular choice for coronary imaging and vessel wall imaging [1]. The strength and flexibility of cardiovascular MR in providing in situ respiratory motion measurements has also permitted the development of a number of more advanced techniques for adapting $k$-space data acquisition with different levels of respiratory induced motion. Despite its success, however, the technique is limited to intra-modality motion management and is difficult to be generalized beyond the MR environment. Nevertheless, the method has demonstrated the value of combining in situ sensing (in this case the movement of the dome of the diaphragm) and a priori subject specific modeling for real-time 3D deformation correction. Thus far, extensive research has also been directed to the use 
of complementary MR-compatible sensing methods to perform real-time measurements of surface distortions particularly due to respiration. The strength of these techniques is that they do not require the interleaved acquisition of navigators, and thus significantly simplify the pulse sequence design. Furthermore, it makes the technique easily transportable to other imaging and therapeutic modalities.

Fig. 1 illustrates a typical setup of using MR compatible surface deformation sensing (in this case by using optical tracker with stereo IR reflectors) for predictive motion modeling. The a priori subject specific motion modeling involves the imaging of the anatomical structure in different deformation states combined with in situ realtime measured sensor outputs. Registration based on free-form deformation or biomechanical modeling can be used to recover the underlying spatio-temporal deformation of the anatomical structure, followed by the estimation of the causality between tissue deformation and real-time sensor signals. At the prediction stage, realtime sensor signals are used to directly predict tissue deformation, thus avoiding the need for further 2D/3D imaging during motion adapted image acquisition or therapy. Such a setup is particularly useful for motion adaptation and attenuation correction of PET imaging which can eliminate the need for a separate and often lengthy PET transmission scan, as well as providing noiseless attenuation correction factors that cannot be achieved by using the current PET approaches.

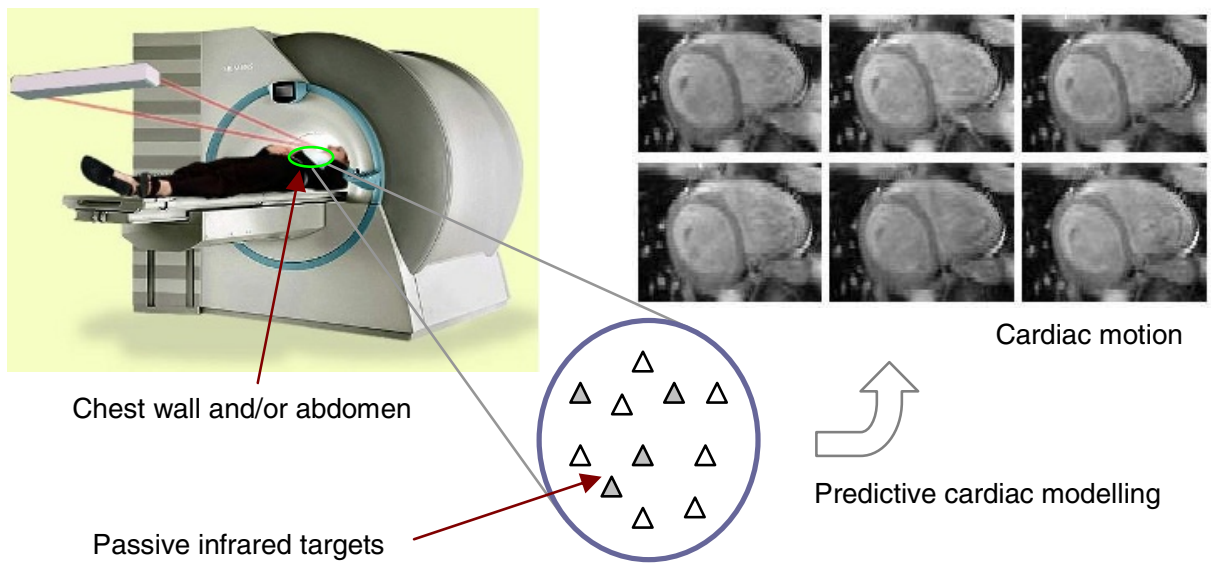

Fig. 1. A systematic illustration of predictive cardiac motion modeling incorporating optimal sensor placement. Not all sensors are sensitive to motion prediction and collinearity is common. The aim of optimal sensor placement is to determine a minimal subset of sensors (shaded) that provides the maximum information content for motion prediction, thus concurrently improving the prediction accuracy and minimizing the sensor usage.

Existing research has shown that surface measured signals for predictive motion modeling can be strongly coupled with each other but poorly correlated with respiratory induced cardiac deformation [2]. This can create significant problems for recovering the inherent model that explains the causality between respiratory motion and surface deformations. Another challenge associated with the technique is in 
optimal sensor placement such that given the number of sensor channels used, the placement of the sensors can provide the maximal information content and model sensitivity to the entire range of tissue deformation. Thus far, the issue of inter-sensor coupling and poor motion correlation has been addressed numerically by the effective use of latent vectors with partial least squares regression or kernel based non-linear regression techniques. Limited research, however, has been directed towards the effect of sensor placement on the accuracy of the motion prediction result. The purpose of this paper is to develop a systematic framework for optimal sensor placement for predictive cardiac motion modeling based on sequential feature selection. We demonstrate that with the proposed method, the number of sensors used can be effectively minimized whilst the prediction accuracy is improved.

\section{Method}

\subsection{Optimal Sensor Placement}

The purpose of optimal sensor placement is to construct and select subsets of features (sensor traces) that can be used to build a good predictor. The nature of the problem is akin to feature selection in machine learning for reducing the complexity of an induction system by eliminating irrelevant and redundant features. For regression learning systems which directly predict continuous value, the task of feature selection can be more difficult because the evaluation criteria based on the properties of discrete classes are inappropriate.

Given a prediction model, the most crucial task is to establish an appropriate feature evaluation criterion to compare each feature subset. For regression, classical criteria are prediction error measures such as RMSEP (root mean square error in prediction) [3] and the RReliefF algorithm as proposed by Marko [4] for estimating the quality of attributes in regression problems. Given candidate features $\left\{x^{l}\right\}, l=1,2, \ldots, M$, the feature subset space can be denoted as $\Omega_{X}$, which is composed of all possible feature combinations $X_{j}$. If we denote the predictive function as $\operatorname{PR}(\cdot)$, the estimated cardiac deformation $\hat{Y}_{j}$ is $\hat{Y}_{j}=\operatorname{PR}\left(X_{j}\right)$ when the input is $X_{j}$. Then the predicted volume $\hat{I}_{j}$ at certain respiratory position can be obtained through wrapping the $3 \mathrm{D}$ volume by the predicted $\hat{Y}_{j}$. When the chosen feature subset is $X_{j}$, the prediction error measure RMSEP is defined as

$$
R M S E P_{j}=\left[\frac{1}{N} \sum_{i=1}^{N}\left(\hat{I}_{j i}-I_{i}\right)^{2}\right]^{1 / 2}
$$

where $N$ is the number of pixels (or voxels) in the region of interest (ROI) for comparison, $I$ is the actually obtained images and $I_{i}, \hat{I}_{j i}$ represent each pixel on the true images and estimated images, respectively. The optimal feature subset $X_{o p}$ can then be chosen as

$$
X_{o p}=\min _{X_{j} \in \Omega_{X}} R M S E P_{j}
$$

and we denote the number of features in $X_{o p}$ as $\left[X_{o p}\right]$. 
Once the evaluation criterion of feature selection has been determined, the next step is to choose a search procedure to explore the space $\Omega_{X}$. Since evaluation criteria are not monotonous, comparison of feature subsets generally amounts to a combinatorial problem (there are $2^{M}-1$ possible subsets for $M$ features), which rapidly becomes computationally impractical even for moderate input sizes. Branch and Bound (BB) [5] feature selection algorithm can be used to reduce the exhaustive search, but it is only feasible with monotonous criteria. Furthermore, the complexity of $\mathrm{BB}$ is still prohibitive for problems with very large feature sets. For these reasons, the following sub-optimal search strategies can be used:

- Sequential forward selection (SFS): it starts with an empty set of features and adds features to the already selected feature set one at a time according to the evaluation criteria [6].

- Sequential backward selection (SBS): it starts with the full set of features and eliminates features from the selected feature set one at a time [7].

- Sequential forward floating selection (SFFS): it includes new features by applying the basic SFS procedure starting from the current feature set, followed by a series of successive conditional exclusion of the worst feature in the newly updated set [8].

- Sequential backward floating selection (SBFS): it excludes new features by applying the basic SBS procedure starting from the current feature set, followed by a series of successive conditional inclusions of the most significant feature from the available features [8].

Floating searching (SFFS and SBFS) is designed to prevent the nesting of feature subsets and also flexibly change the fixed values of $l$ and $r$ in the Plus $l$-Take away $r$ algorithm to approximate the optimal solution as much as possible.

\subsection{Predictive Motion Modeling}

To recover cardiac deformation and establish its intrinsic correlation with real-time measurable surface signals, 3D image volumes depicting different stages of the cardiac deformation due to respiration are used. In this study, predictive motion modeling and correction is achieved by partial least squares regression (PLSR). Specifically, PLSR searches for a set of components (called latent vectors or score vectors) that perform a simultaneous decomposition of $\mathrm{X}$ and $\mathrm{Y}$ with the constraint that these components explain as much as possible of the covariance between $\mathrm{X}$ and $\mathrm{Y}$. It is followed by a regression step where the decomposition of $\mathrm{X}$ is used to predict $\mathrm{Y}$. The simultaneous decomposition of $\mathrm{X}$ and $\mathrm{Y}$ is

$$
\begin{aligned}
& \mathrm{X}=\mathrm{TP}^{\mathrm{T}}+\mathrm{E} \\
& \mathrm{Y}=\mathrm{UQ}^{\mathrm{T}}+\mathrm{F}
\end{aligned}
$$

where $\mathrm{T}$ and $\mathrm{U}$ are $(N \times k)$ matrices of the extracted $k$ score vectors, the $(p \times k)$ matrix $\mathrm{P}$ is the factor loading matrix, the $(q \times k)$ matrix $\mathrm{Q}$ is the coefficient loading matrix, and the $(N \times p)$ matrix $\mathrm{E}$ and the $(N \times q)$ matrix $\mathrm{F}$ are residuals. PLSR 
tries to find a score vector $\mathrm{t}$ in the column space of $\mathrm{X}$ and a score vector $\mathrm{u}$ in the column space of $\mathrm{Y}$ with the constraints that $\|\mathrm{w}\|=1,\|\mathrm{q}\|=1$ such that

$$
\begin{aligned}
& \mathrm{t}=\mathrm{Xw} \\
& \mathrm{u}=\mathrm{Yq}
\end{aligned}
$$

to give the maximal squared covariance for $\left(\mathrm{u}^{\mathrm{T}} \mathrm{t}\right)^{2}$

$$
\max [\operatorname{cov}(\mathrm{t}, \mathrm{u})]^{2}=\max _{\|\mathrm{w}\|=\|\mathrm{qq}\|=1}[\operatorname{cov}(\mathrm{Xw}, \mathrm{Yq})]^{2} .
$$

Rather than linking measurements $\mathrm{X}$ and $\mathrm{Y}$ directly, PLSR tries to establish the inner relationships between $\mathrm{T}$ and $\mathrm{U}$. A linear model is assumed to relate the score vectors

$$
\mathrm{U}=\mathrm{TD}+\mathrm{H}
$$

where $\mathrm{D}$ is a diagonal matrix that has the regression weights as the diagonal elements, and $\mathrm{H}$ denotes residuals similar to $\mathrm{E}$ and $\mathrm{F}$ above. This allows $\mathrm{Y}$ to be modeled by $\mathrm{T}$ and $\mathrm{Q}$ as

$$
\mathrm{Y}=\mathrm{TQ}^{\mathrm{T}} \mathrm{D}+\mathrm{F}^{*} .
$$

Numerically, the classical solution to the equations presented above is based on the nonlinear iterative partial least squares (NIPALS) algorithm [9].

\subsection{Validation}

To extract subject specific respiratory induced cardiac deformation patterns, ten normal subjects were recruited and underwent MR imaging on a Siemens Sonata 1.5T MR scanner. After scout scans, a segmented 3D TrueFISP sequence was used to acquire short-axis image volumes in free breathing with navigator-echoes and oversampling. The 3D stack comprised of 14 short axis slices from the valve plane to the apex throughout the full respiratory range. Data acquisition was repeated 20 times for total acquisition duration of 560 cardiac cycles. The imaging parameters used include an RF flip angle of $65^{\circ}$, in plane matrix size of $256 \times 102$, pixel size of $1.56 \mathrm{~mm} \times$ $2.70 \mathrm{~mm} \times 8.78 \mathrm{~mm}$, and FOV of $400 \mathrm{~mm} \times 275 \mathrm{~mm} \times 123 \mathrm{~mm}$.

All 3D datasets were generated at different respiratory positions by retrospective respiratory gating. In order to reconstruct a 3D data set for a given respiratory position, each segment contributing to the data set was chosen from the over-sampled data when the accompanying navigator echo yielded a diaphragm position nearest to the given respiratory level. For each subject, a total of six and seven volumes covering different respiratory positions from end-inspiration to end-expiration were created.

Sixteen surface traces lying on the chest wall were placed in a $4 \times 4$ grid with 25 $\mathrm{mm}$ spacing. The end-expiration volume was selected as the reference volume to which the other volumes were registered with free-form deformation to recover the underlying spatio-temporal deformation field of the cardiac structure. The deformation of each volume was characterized by the movement of control vertices of the B-spines. The intrinsic relationship between tissue deformation and real-time measurable signals associated with different phases of respiratory motion was then 
extracted through the motion prediction technique described above. Sequential selections based on SFS, SBS, SFFS, and SBFS were used to determine the optimal sensor placement and its associated prediction errors. The prediction errors of the optimal feature subset obtained from above four sequential search procedures were compared. To obtain an unbiased assessment of the performance of each feature subset for predicting cardiac motion, a leave-one-out cross-validation was performed on all image volumes and the RMSEP for each volume was normalized against the registration results. Finally, the averaged RMSEP was used as the quality measure.

\section{Results}

By the use of the first two significant latent vectors for PLSR analysis, Fig. 2(a) illustrates the relative performance of SFS, SBS, SFFS and SBFS for determining the optimal sensor placement for one of the subjects studied, where a small value

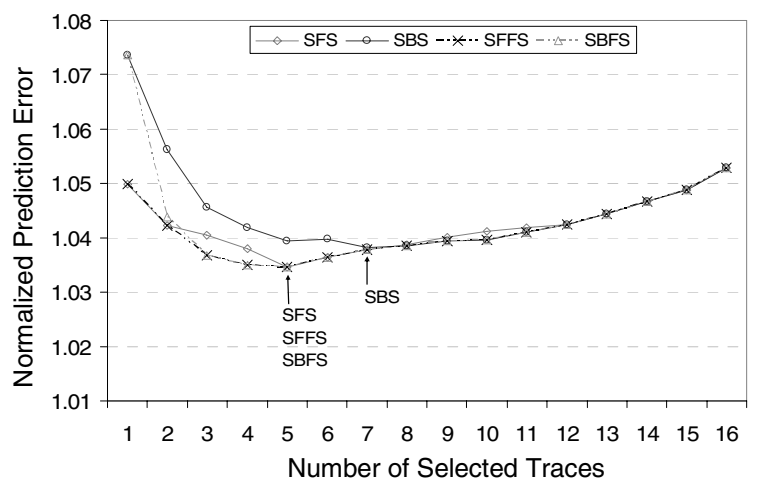

(a)

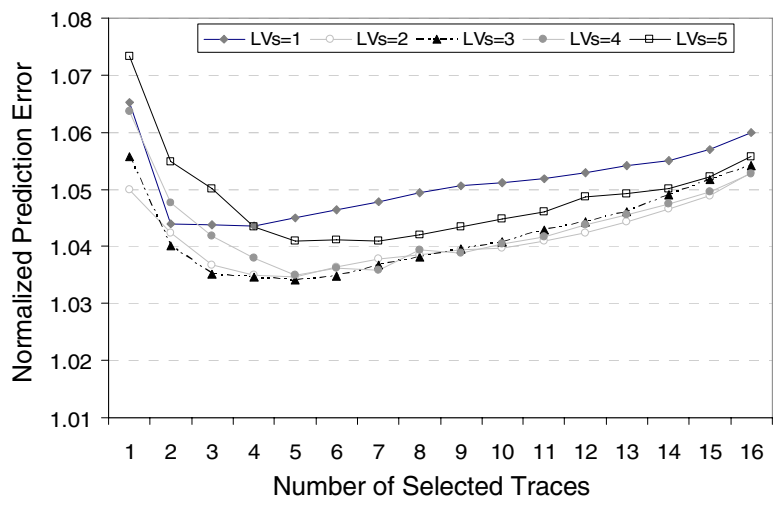

(b)

Fig. 2. (a) Comparison of SFS, SBS, SFFS and SBFS searching procedures for optimal sensor placement, where the number of optimal sensors derived from each technique is indicated by an arrow. (b) The performance of SFFS algorithm when different number of latent vectors is chosen for the PLSR prediction model. 
Table 1. Quantitative assessment of the feature selection algorithms on ten subjects with the number of optimal traces and corresponding normalized RMSEP

\begin{tabular}{c|c|cc|cc|cc|cc}
\hline & 16 & \multicolumn{9}{|c}{ Feature selection algorithm } \\
\cline { 3 - 9 } Case & traces & \multicolumn{2}{|c|}{ SFS } & \multicolumn{2}{c}{ SBS } & \multicolumn{2}{c}{ SFFS } & \multicolumn{2}{c}{ SBFS } \\
& RMSEP & RMSEP & {$\left[X_{o p}\right]$} & RMSEP & {$\left[X_{o p}\right]$} & RMSEP & {$\left[X_{o p}\right]$} & RMSEP & {$\left[X_{o p}\right]$} \\
\hline 1 & 1.0452 & 1.0245 & 3 & 1.0270 & 6 & 1.0245 & 3 & 1.0245 & 3 \\
2 & 1.3574 & 1.1668 & 3 & 1.1571 & 5 & 1.1571 & 5 & 1.1571 & 5 \\
3 & 1.0439 & 1.0356 & 4 & 1.0352 & 7 & 1.0351 & 6 & 1.0356 & 5 \\
4 & 1.0223 & 1.0191 & 6 & 1.0201 & 8 & 1.0191 & 6 & 1.0200 & 7 \\
5 & 1.0528 & 1.0346 & 5 & 1.0383 & 7 & 1.0346 & 5 & 1.0346 & 5 \\
6 & 1.0368 & 1.0228 & 9 & 1.0227 & 10 & 1.0227 & 10 & 1.0227 & 10 \\
7 & 1.0858 & 1.0568 & 2 & 1.0591 & 4 & 1.0568 & 2 & 1.0568 & 2 \\
8 & 1.0823 & 1.0659 & 4 & 1.0661 & 6 & 1.0659 & 4 & 1.0661 & 6 \\
9 & 1.0552 & 1.0504 & 8 & 1.0489 & 7 & 1.0489 & 7 & 1.0489 & 7 \\
10 & 1.0569 & 1.0328 & 3 & 1.0328 & 3 & 1.0328 & 3 & 1.0328 & 3 \\
\hline
\end{tabular}

represents a good motion prediction result. It is evident from Fig. 2(a) that the optimal number of sensors is 5, and SFS, SFFS, and SBFS seem to provide similar results. This figure also reveals an important fact that as the number of sensors increases, the quality of the prediction result in fact deteriorates. This performance drop can be attributed to a number of factors, but intrinsic errors and non-linearity of individual sensors across the entire respiratory range are the main causes of concern. Fig. 2(b) illustrates the model dependence of the proposed feature selection algorithms when different model parameters are chosen for prediction. It highlights the importance of optimal sensor placement for the practical deployment of predictive motion management techniques, which has so far only been handled in an ad hoc manner.

In order to provide an overview of the predictive errors for the ten subjects studied, Table 1 lists the normalized RMSEP for each of the sequential selection algorithms used and the optimal number of sensors to be used. It has been shown that for all the subjects studied, the accuracy of predictive cardiac modeling has been greatly increased after optimal sensor selection. The average number of minimal sensor placement is about 5, which is significantly lower than the original 16 channels used. Compared with the traditional SFS and SBS sequential procedure, the floating searching strategies seem to provide better overall results.

\section{Discussion and Conclusion}

In this paper, we have developed a systematic framework of predictive cardiac motion modeling with optimal sensor placement. Subject specific respiratory patterns are a significant problem for motion management in in vivo imaging. The proposed framework effectively resolves the problem encountered by traditional regression methods in that we used the latent variables from both the input and output of the regression model to establish their inner relationships. This study demonstrates that the use of optimal sensor placement not only minimizes the sensing channels but also improves the overall prediction accuracy. Although in MR the use of navigator echoes 
has already improved the quality of the images acquired, particularly for capturing small mobile structures such as the coronaries, its wide spread use is still hindered by its lack of general adaptivity to different imaging platforms. The results from this study suggest that being effectively a real-time technique, the proposed method is not only reasonably accurate, but also able to predict respiratory induced cardiac motion from purely surface measurable signals. The development of a general method for 3D motion prediction based on easily measured surface signals will have significant impact on other parallel imaging modalities and the development of new integrated imaging modalities such as PET/MR and PET/CT.

\section{References}

1. J. Keegan, P.D. Gatehouse, G.Z. Yang, D.N. Firmin., "Coronary Artery Motion with the Respiratory Cycle during Breath-holding and Free-breathing: Implications for Slicefollowed Coronary Artery Imaging," Magn Reson Med., Vol. 47, pp. 476-481, 2002.

2. N. Ablitt, J. Gao, J. Keegan, L. Stegger, D.N. Firmin, G.Z. Yang, "Predictive Cardiac Motion Modeling and Correction with Partial Least Squares Regression," IEEE Trans. Med. Imag., Vol. 23, pp. 1315-1324, Oct. 2004.

3. R. Leardi, A.L. González, "Genetic Algorithms Applied to Feature Selection in PLS Regression: How and When to Use Them," Chemometrics and Intellingent Laboratory Systems, Vol. 41, pp. 195-207, 1998.

4. Marko Robnik-Sikonja, Igor Kononenko, "An Adaptation of Relief for Attribute Estimation in Regression," In D.Fisher (ed.): Machine Learning, Proceedings of 14th International Conference on Machine Learning ICML'97, Nashville, TN, 1997.

5. P.M. Narendra, K. Fukunaga, "A Branch and Bound Algorithm for Feature Subset Selection," IEEE Trans. Comput., Vol. 26, pp. 917-922, 1977.

6. A.W. Whitney, "A Direct Method of Nonparametric Measurement Selection," IEEE Trans. Comput., Vol. 20, pp. 1100-1103, 1971.

7. T. Marill, D.M. Green, "On the Effectiveness of Receptors in Recognition System," IEEE Trans. Inform. Theory, Vol. 9, pp. 11-17, 1963.

8. P. Pudil, J. Novovicova, J. Kittler, "Floating Search Methods in Feature Selection," Pattern Recognition Letters, Vol. 15, pp. 1119-1125, Nov. 1994.

9. H.Wold. Soft Modeling by Latent Variables: the Nonlinear Iterative Partial Least Squares Approach. In J. Gani, editor, Perspectives in Probability and Statistics, pp. 520-540. Academic Press, London, 1975. 